Article Type: Research Paper

\title{
MEASURING CONTAGION RISK ON BANKING SYSTEM IN THE DIGITAL ERA
}

\author{
Musdholifah*, Ulil Hartono, and Yulita Wulandari
}

\section{마료}

\section{AFFILIATION:}

Universitas Negeri Surabaya, Surabaya, Indonesia.

*CORRESPONDENCE:

musdholifah@unesa.ac.id

THIS ARTICLE IS AVALILABLE IN:

http://journal.umy.ac.id/index.php/esp

DOI: $10.18196 /$ jesp.20.2.5025

\section{CITATION:}

Musdholifah, Hartono, U., \& Wulandari, Y. (2019). Measuring Contagion Risk on Banking System in The Digital Era. Jurnal Ekonomi \& Studi Pembangunan, 20(2), 207220.

\section{ARTICLE HISTORY}

Received:

24 August 2019

Accepted:

29 October 2019

\begin{abstract}
As an essential institution to the practice of national payment flow, banks always confront with various risk exposures inherent in them. An interbank interactions through interbank money market might yield higher systemic risks that can lead to a default. This study aims at determining the contagion effects towards Indonesian banks. This study used 18 bank samples who provided annual reports from 2007 to 2016. The measurement of the systemic risks was performed by using financial contagion risk index and was tested using Vector Autoregression method. Results show that there was a one-way causality pattern between banks as the research samples, covering BCA with Bank Mayapada, Bank Maybank, Bank Mega, and Bank Resona Perdania and also Bank CIMB Niaga with BCA, BRI, BNI, BTN, Commonwealth Bank, J-Trust Bank, Bank KEB Hana, Bank Mega, and Bank Permata. Meanwhile, two-way causality occurs between Bank BCA and Bank Mandiri and vice versa. In addition, the impact of the risk pressure of a bank is not always positive, however, it is also negative in some cases, which means that the bank can take advantage of the shocked conditions experienced by other banks.
\end{abstract}

Keywords: Contagion; Systemic Risk; Interbank Market; Banking Institution; Shock.

JEL Classification: G21; G01

\section{Introduction}

Banks have an important position in the national economic system. Its role is getting broader with a variety of services offered that provides convenience to the public, especially in case of financial matters. Moreover, a bank also has various risks inherent in it. In coping with the banking crisis occurred, banks become a flimsy institution due to the various exposures they have. Financial institutions have an exposure effect towards each other that can be seen from the practice of interbank market. Meaning that, a failure occurred in one institution will be contagious and cause further failures in other institutions which run similar business models (Kapoor, 2010; 19-20).

Ayomi and Hermanto (2013) explain that systemic risk causes failure of one or several financial institutions as a result of systemic events due to financial system imperfections such as asymmetric information, agency 


\section{Musdholifah, Hartono, \& Wulandari \\ Measuring Contagion Risk on Banking System in The Digital Era}

problems, and moral hazard. Those things will cause excessive risk-taking behavior, contagion risk (domino effect), and financial intermediation.

Zakaria (2015) explains that the contagion effects can come from finance and economics. Financial contagion occurs because the agents in domestic and international financial systems are interconnected through the transactions in the financial market, either directly or indirectly. One condition that triggers systemic risk is bank run. Simorangkir (2011) defines bank run as an event in which many customers simultaneously withdraw funds on a large scale conducted as soon as possible in a certain bank because the customer does not longer believe the bank's performance. Consequently, this condition might turn into a banking crisis if the bank run occurred in one bank has been transmitted to another bank, known as a contagious effect. Schoenmaker (1996) defines banking contagion risk as the condition of financial difficulties in one or more banks that are transmitted to a number of other larger banks or financial systems. The contagion effect can spread through information or credit channels.

Some researchers have undertaken research on banking contagion effects. Aharony and Swary (1983) examined the possibility of a contagion effect using capital market when a bank failure in the US occurred. The results show that there is little evidence of the contagion due to the failure of three major banks in the US. It happened because during the transmission period, the central bank played an active role as a lender of the last resort so that the impact of transmission failure can be reduced. This caused doubts for banking experts leading to the idea that the contagion effect had no significant impact towards banking stability.

Meanwhile, Zakaria (2015) conveys that systemic risk is a big source of risk. There were banks in Morocco have potential systemic problems, such as ATW and BMCE. Grais and Rajhi (2015) also prove that Islamic financial institutions could potentially experience the contagion risk in different degrees. The Islamic financial institutions face certain risks related to Sharia principles in their business activities. Billio, Getmansky, Lo, and Pelizzon (2010) explain that the banking sector can be a source of systemic risk compared to other financial institutions. Bank liquidity and the fact that the banking sector is not designed to withstand large and rapid losses, make this sector vulnerable to systemic risk. Gauthier, $\mathrm{He}$, and Souissi (2010) used bank liquidity to see its effect towards bank systemic risk and found that the network effects and liquidity risk were the main causes of systemic risk in the banking system.

Moreover, Upper (2011) describes the simulation method used to predict the potential contagion found in the interbank loan market. Similarly, Chinazzi and Fagiolo (2013) used a network theory to observe the contagion and systemic risk in the financial markets. Results show that the relationship or interaction between banks and financial institutions can strengthen the shock of the economic system during the crisis period.

Based on the description, this study aims at determining the causal relationship between banks in Indonesia and examine whether shocks in a bank will cause similar effects to 


\section{Musdholifah, Hartono, \& Wulandari \\ Measuring Contagion Risk on Banking System in The Digital Era}

other banks within the banking system in Indonesia. The study was conducted using the Granger's causality test method.

Globalization leads to the connections between financial institutions and money markets both domestically and internationally. This causes the collapse of a financial institution in one country, which might spread to other institutions or countries (Shah, 1997), of which the phenomenon is often called as contagion or systemic risk. In the crisis period, the probability of default from several financial institutions will increase the default on other financial institutions. It means that when financial institutions experience distress, other similar institutions will experience shocks, which is further contagious (Zakaria, 2015). Such phenomenon is similar to the domino effect because the fall of an institution leads to the fall of other institutions in a row. On a broader scale, the collapse of a certain bank in a country will cause stress to the banking institutions and other financial institutions in other countries. It can be seen in the 2008 US crisis which later spread becoming global financial crisis.

Rescue costs for banks that experience stressed conditions in preventing the systemic collapse are quite large. Shah (1997) states that these costs can reach millions to billions of pounds, so there is a need for research in this field as an effort to prevent the occurrence of systemic risk transmission. There are several studies that have been conducted to determine the cause of the contagion effect. Most researchers realize that the interbank market is the most important risk distribution channel for banks and other financial institutions (Memmel \& Sachs, 2013). Moreover, the distribution channel can also occur in the context of liquidity and refinancing (Schoenmaker, 1996).

One of the studies examining transmission of shocks from the interbank market side was conducted by Philippas, Koutelidakis, \& Leontitsis (2015) who adapted the Barabási Albert model (BA model). The shocks in small banks caused huge losses in a whole so that there was a need for a crisis restraint policy. The interbank networks can be used to explain the shocks and the correlation between the banking sectors.

Gai, Haldane, and Kapadia (2011) and Gauthier, et al. (2010) empirically examined the distribution channel from a banking crisis in terms of the liquidity. Gai, et al. (2011) developed an interaction network model in order to find out how the channel of liquidity shock spread in the banking system. The results show that the concentration and complexity are the main causes of the fragility of the financial institutions until they are able to be the channel which spread shocks in the economic cycle.

In general, Aharony and Swary (1983) distinguish the spread of contagion effects through information and credit channels. The spread of contagion effects through information channels is divided into pure and noisy contagion. Pure contagion occurs when negative information such as fraud and investment losses on certain risks that occur in a bank adversely affect all banks, including the banks that have no connection with the first bank. Noisy contagion occurs when a bank's failure shows a bad signal for some other banks with similar characteristics. If a bank fails, the other banks with similar asset structures and liabilities will also likely experience bank runs. Meanwhile, the spread of contagion 
effects through credit channels can occur due to the existence of interbank markets, overthe-counter (OTC) derivative markets, and payment systems.

The spread of contagion risk can also occur through foreign exchange transactions in the market and the fair value of financial assets (De Bandt \& Hartmann, 2000). It is because the foreign exchange and security transactions have two sides, namely the transfer of assets on one hand and payments on the other, which both lead to the exposure of credit and liquidity risks. The research framework in this study refers to the previous study conducted by Christiawan and Arfianto (2013), which had similar subjects and research methods. Therefore, based on the previous research and literary objectives, the hypotheses proposed in this study are stated as follows:

H1 : There is a causal relationship between interbank banking pressures

H2 :There is an effect of shocks on bank $i$ towards bank $j$

\section{Research Method}

This study aimed at determining the contagion risk in several banks in Indonesia. The calculation of the contagion risk was done through the use of composite index taking three variables into account:

\section{The comparison between the placements to other banks and deposit}

This variable indicated that the banks had account interactions with other banks (interbank deposits) so that this indicator could be used to measure the bank's vulnerability towards the contagion risk in the interbank market. The formula is:

$$
\frac{\text { placements to other banks }}{\text { deposit }}
$$

\section{The differences between the increase of fair-value financial asset and the total assets}

This variable was used to assess the resilience of the bank through the total assets towards the shocks that occurred in the securities market. The formula is:

$$
\frac{\text { differences between the increase of fair - value financial asset }}{\text { total asset }}
$$

\section{The differences between foreign exchange transactions and the total assets}

This variable was used to measure the bank's resilience through its total assets in responding to the shocks on the foreign exchange market. The formula is: 


\section{Musdholifah, Hartono, \& Wulandari \\ Measuring Contagion Risk on Banking System in The Digital Era}

The differences between foreign exchange transactions

total asset

Those three variables were used to measure the financial contagion risk indicator (Christiawan \& Arfianto, 2013). The indicator was in a form of an index used to measure the systemic risk between banks and the channels of transmission through the interbank money market, foreign exchange transactions, and the fair value of financial assets in the banks. The research population covered all conventional banks in Indonesia. Meanwhile, the samples used in this study were banks that provided financial reports from 2007-2016. Therefore, based on that characteristic, there were 18 banks taken as the research samples. The data used in this study were audited financial statements and were available on each bank's website or on the website of Indonesian Stock Exchange.

The method used in the present study was Vector Autoregression (VAR). An analysis with the VAR method was carried out in sequence to answer the research hypotheses. Simple VAR equation were presented as:

$$
\begin{aligned}
& y_{1 t}=\beta_{10}+\beta_{11} y_{1 t-1}+\alpha_{11} y_{2 t-1}+u_{1 t} \\
& y_{2 t}=\beta_{20}+\beta_{21} y_{2 t-1}+\alpha_{21} y_{1 t-1}+u_{2 t}
\end{aligned}
$$

In Matrix form VAR equation were

$$
\begin{gathered}
\left(\begin{array}{l}
y_{1 t} \\
y_{2 t}
\end{array}\right)=\left(\begin{array}{l}
\beta_{10} \\
\beta_{20}
\end{array}\right)+\left(\begin{array}{l}
\beta_{11} \alpha_{11} \\
\alpha_{21} \beta_{21}
\end{array}\right)+\left(\begin{array}{l}
y_{1 t-1} \\
y_{2 t-1}
\end{array}\right)= \\
\left.\underset{2 \mathrm{x} 1}{y_{t}}=\underset{2 \times 1}{\beta_{0}}+\underset{(2 \times 2)(2 \times 1)}{\beta_{1} y_{t-1}}+\underset{(2 \times 1)}{u_{1 t}} u_{2 t}\right)
\end{gathered}
$$

In order to answer the first hypothesis, the Granger's causality test was conducted. The proposed $\mathrm{HO}$ stated that there was no causal relationship between interbank pressures, while Ha showed the contrary. $\mathrm{HO}$ was rejected if the probability value was smaller than the real values. To answer the second hypothesis, VAR test was conducted to see the effect of shocks from one bank to other banks.

\section{Result and Discussion}

\section{Granger's Causality Test}

To answer the first hypothesis, the granger Causality test was carried out by using $\alpha$ of $5 \%$, showing a result that there was a causal relationship between banks in both one-way and two-way causalities. Statistically, one-way causality occurred between Bank CIMB Niaga which affected BTN, BCA, BRI, BNI, Commonwealth Bank Indonesia, Bank Permata, Bank Mega, KEB Hana Bank, and J-Trust Indonesia Bank. Meanwhile, Bank BCA influenced Bank Mayapada International, Maybank Indonesia, Bank Mega, and Bank Resona 


\author{
Musdholifah, Hartono, \& Wulandari \\ Measuring Contagion Risk on Banking System in The Digital Era
}

Perdania. BRI influenced BNI, Bank Ekspor Indonesia, Mayapada Bank, and Bank Nusantara Parahyangan. Bank Mandiri affected BRI, BNI, and Commonwealth Indonesia. Bank Commowealth Indonesia influenced BNI and Bank Mega. BTN affected BCA. BNI affected Bank Danamon Indonesia. J-Trust Bank affected BNI. Mayapada International Bank affected BNI. Bank Permata influenced Bank Mayapada International and BNI. Bank Woori Saudara Indonesia influenced BNI. Finally, Bank Ekspor Indonesia affected Maybank Indonesia and Bank Resona Perdania. In addition, the results of the granger test also showed that, statically, there was a two-way relationship or causality between BCA and Bank Mandiri.

\title{
Vector Auto Regression Analysis
}

Based on the granger causality test, it could be seen that there was an interbank causality in both one-way and two-way causalities. Next, a VAR analysis was conducted to determine the impact of shocks from bank $j$ towards bank i. Although it was statistically significant, the impact of shocks was not always positive. Based on the results of the tests, there were many cases indicating that the impact of the shock was negative, which meant that bank $\mathrm{i}$ could take advantage of the shock that occurred in bank $\mathrm{j}$.

Large banks had large asset values so that they could reduce the risk of the shock impacts that occurred in other banks. In some cases, the shock of other banks gave benefits to the aforementioned banks. One of the banks with large assets was BNI. Based on the results of the VAR analysis, if the risk in BCA four periods ago changed by $1 \%$, it could reduce BNI's risk in this period by $2.78837 \%$. Moreover, if the BTN risk three periods ago increased by $1 \%$, it would reduce BNI's risk at this period by $2.09282 \%$. The same thing happened with the increase in Bank Danamon's risk in the last period, Bank Ekspor Indonesia a period ago and three periods ago, Indonesian J-Trust Bank one period and three periods ago, KEB Hana Bank one period ago and three periods ago, Bank Mayapada two periods ago and four periods ago, Maybank Bank last period and three periods ago, Bank Mega four periods ago, Bank Nusantara Parahyangan two periods ago and four periods ago, Bank Permata last period and three periods ago, and Bank Resona Perdania two periods ago and four periods ago; would statistically reduce BNI's risk by a percentage reflected in Table 1 (Appendix).

The Bank shocks could also be caused by the condition of the bank in the past. As an example, for BNI, the shock occurring last period, two periods ago, and three periods ago, led to the shocks in the current period. BCA, as one of the national private banks with large assets, also experienced shocks due to the past conditions. In several banks with not-too-large assets, the effects of shocks did not cause significant shock to other banks. An example could be seen from Bank Mayapada International in which there were not many banks affected by the shock. In addition, the interbank shocks, even though they were statistically positive, were not too significant because the shock did not directly cause default conditions to other banks. This condition also did not cause a systemic crisis in banks in Indonesia. It supported a research conducted by Zakaria (2015) which stated that contagion occurred due to the agents in financial systems that were connected through financial market transactions directly or indirectly. This was also in line with the 
results of the research done by Christiawan and Arfianto (2013) showing that there was interbank causality, and the impact of shocks did not directly cause complacency to other banks. The limitation of this study was that it only dealt with the contagion risk in terms of the market risk as stated in the researches done by Memmel \& Sachs (2013) and Philippas, et al. (2015), and also it did not take the contagion risk into account in terms of the liquidity as stated in Gai, Jenkinson, \& Kapadia (2007), Gai, et al. (2011), Gauthier, et al. (2010), Allen and Gale (2000), Allen, Carletti, and Gale, (2009), Chen, Chen, \& Gerlach (2013), Ahelegbey and Giudici (2014).

\section{Conclusion}

This study aims at analyzing the pressures and the impacts of the shock effect from one bank to other banks. The spread of the contagion risk in this study was measured from the interbank market risk channels by taking the bank vulnerabilities in interbank account interactions, the risks of the securities market and foreign exchange market. The test results show that there is one-way and two-way causalities, for two-way relationship or causality it found that there is causality between BCA and Bank Mandiri. In addition, there is also an impact of the shock from banks $j$ towards bank $i$, of which the statistics are not always positive. This shows that there is pressure among the banks in Indonesia. Although statistically there are inter-bank shocks, the shock value tends to be small so that it does not lead to direct systemic crisis. The limitation in this study is that it does not take the distribution channel in terms of liquidity, therefore, the future studies are expected to take the contagion risk assessment in terms of liquidity into account.

\section{Acknowledgment}

This research was supported by Ministry of Research, Technology and Higher Education. We thank our colleagues from Unesa who provided insight and expertise that greatly assisted the research.

\section{Appendix}

Table 1 VAR Analysis

\begin{tabular}{|c|c|c|c|c|c|c|c|c|}
\hline Bank & Bank & Respon & Bank & Bank & Respon & Bank & Bank & Respon \\
\hline \multirow[t]{10}{*}{ BCA } & $\mathrm{BCA}(-2)$ & -3.54 & \multirow[t]{10}{*}{$\mathrm{BNI}$} & $\mathrm{BCA}(-4)$ & -2.79 & \multirow[t]{10}{*}{$B R I$} & $\mathrm{BCA}(-2)$ & -2.36 \\
\hline & $\mathrm{BCA}(-3)$ & -3.25 & & BNI (-1) & -2.78 & & $\mathrm{BCA}(-3)$ & -2.91 \\
\hline & BRI (-1) & 1.84 & & BNI (-2) & -3.53 & & BNI (-1) & -2.14 \\
\hline & BRI (-2) & -3.51 & & BNI (-3) & -3.2 & & BRI (-2) & -2.54 \\
\hline & BRI (-3) & -2.68 & & BRI (-2) & 4.33 & & BRI (-4) & -1.88 \\
\hline & BRI (-4) & -2.52 & & BRI (-3) & 4.79 & & BTN (-1) & 5.97 \\
\hline & BTN (-1) & 5.76 & & BTN (-3) & -2.09 & & BTN (-4) & -4.19 \\
\hline & BTN (-2) & -2.09 & & $\begin{array}{l}\text { Danamon (- } \\
\text { 1) }\end{array}$ & -2.04 & & $\begin{array}{l}\text { CIMB Niaga } \\
(-1)\end{array}$ & 4.74 \\
\hline & BTN (-4) & -4.01 & & $\begin{array}{l}\text { Ekspor_Ind (- } \\
\text { 1) }\end{array}$ & -2.6 & & $\begin{array}{l}\text { CIMB Niaga } \\
(-2)\end{array}$ & 3.63 \\
\hline & $\begin{array}{l}\text { CIMB Niaga } \\
(-1)\end{array}$ & 4.10 & & $\begin{array}{l}\text { Ekspor_Ind (- } \\
\text { 2) }\end{array}$ & 2.71 & & $\begin{array}{l}\text { CIMB Niaga } \\
(-3)\end{array}$ & 3.1 \\
\hline
\end{tabular}


Musdholifah, Hartono, \& Wulandari

Measuring Contagion Risk on Banking System in The Digital Era

\begin{tabular}{|c|c|c|c|c|c|c|c|c|}
\hline Bank & Bank & Respon & Bank & Bank & Respon & Bank & Bank & Respon \\
\hline \multirow[t]{10}{*}{$\mathrm{BCA}$} & $\begin{array}{l}\text { CIMB Niaga (- } \\
\text { 2) }\end{array}$ & 3.48 & & $\begin{array}{l}\text { Ekspor_Ind } \\
(-3)\end{array}$ & -2.77 & \multirow[t]{2}{*}{ BTN } & Mega (-1) & 2.27 \\
\hline & $\begin{array}{l}\text { CIMB Niaga (- } \\
\text { 3) }\end{array}$ & 2.86 & & $\begin{array}{l}\text { Ekspor_Ind } \\
(-4)\end{array}$ & 2.76 & & Mega (-2) & -2.02 \\
\hline & Danamon (-2) & -2.24 & & $\begin{array}{l}\text { Jtrust_Ind } \\
(-1)\end{array}$ & -2.65 & & Mega (-3) & 1.71 \\
\hline & $\begin{array}{l}\text { Ekspor_Ind (- } \\
\text { 1) }\end{array}$ & 2.01 & & $\begin{array}{l}\text { Jtrust_Ind } \\
(-2)\end{array}$ & 2.73 & & $\begin{array}{l}\text { Resona_Perd } \\
\text { ania }(-1)\end{array}$ & 2.24 \\
\hline & $\begin{array}{l}\text { Ekspor_Ind (- } \\
\text { 2) }\end{array}$ & -1.95 & & $\begin{array}{l}\text { Jtrust_Ind } \\
(-3)\end{array}$ & -2.74 & & $\begin{array}{l}\text { Woori_SDRA } \\
(-1)\end{array}$ & 1.8 \\
\hline & $\begin{array}{l}\text { Ekspor_Ind (- } \\
\text { 3) }\end{array}$ & 1.92 & & $\begin{array}{l}\text { Jtrust_Ind } \\
(-4)\end{array}$ & 2.7 & & $\begin{array}{l}\text { Woori_SDRA } \\
(-2)\end{array}$ & -1.93 \\
\hline & $\begin{array}{l}\text { Ekspor_Ind (- } \\
\text { 4) }\end{array}$ & -1.93 & & $\begin{array}{l}\text { KEBHana (- } \\
\text { 1) }\end{array}$ & -2.94 & & $\begin{array}{l}\text { Woori_SDRA } \\
(-3)\end{array}$ & 2.00 \\
\hline & Mandiri (-4) & -1.82 & & $\begin{array}{l}\text { KEBHana (- } \\
\text { 2) }\end{array}$ & 2.94 & & $\begin{array}{l}\text { Woori_SDRA } \\
(-4)\end{array}$ & -2.09 \\
\hline & Maybank (-1) & 2.07 & & $\begin{array}{l}\text { KEBHana (- } \\
\text { 3) }\end{array}$ & -2.88 & & BCA (-2) & 2.12 \\
\hline & Maybank (-2) & -2.05 & & $\begin{array}{l}\text { KEBHana (- } \\
\text { 4) }\end{array}$ & 2.76 & & $\mathrm{BCA}(-3)$ & 2.99 \\
\hline \multirow{21}{*}{$\begin{array}{l}\text { COMMO } \\
\mathrm{N} \\
\bar{H}_{\mathrm{H}} \text { WEALT }\end{array}$} & $\mathrm{BCA}(-2)$ & -2.16 & & $\begin{array}{l}\text { Nusantara_ } \\
\text { Parahy }(-4)\end{array}$ & -2.61 & & KEBHana $(-1)$ & -1.92 \\
\hline & $\mathrm{BCA}(-3)$ & -2.94 & & $\begin{array}{l}\text { Permata (- } \\
\text { 1) }\end{array}$ & -2.77 & & KEBHana (-2) & 2.28 \\
\hline & BNI (-1) & 1.98 & & $\begin{array}{l}\text { Permata (- } \\
\text { 2) }\end{array}$ & 2.84 & & KEBHana (-3) & -2.39 \\
\hline & BRI (-2) & -3.02 & & $\begin{array}{l}\text { Permata (- } \\
\text { 3) }\end{array}$ & -2.79 & & KEBHana (-4) & 2.42 \\
\hline & BRI (-3) & -2.89 & & $\begin{array}{l}\text { Permata (- } \\
\text { 4) }\end{array}$ & 2.71 & & Mandiri (-4) & 1.9 \\
\hline & BTN (-1) & 2.94 & & $\begin{array}{l}\text { Resona_Per } \\
\text { dania }(-1)\end{array}$ & 1.90 & & $\begin{array}{l}\text { Mayapada (- } \\
\text { 3) }\end{array}$ & 1.86 \\
\hline & BTN (-4) & -2.47 & & $\begin{array}{l}\text { Resona_Per } \\
\text { dania }(-2)\end{array}$ & -2.57 & & $\begin{array}{l}\text { Mayapada (- } \\
\text { 4) }\end{array}$ & -2.09 \\
\hline & $\begin{array}{l}\text { Ekspor_Ind (- } \\
\text { 1) }\end{array}$ & 2.26 & & $\begin{array}{l}\text { Resona_Per } \\
\text { dania }(-3)\end{array}$ & 2.53 & & Maybank (-1) & -2.45 \\
\hline & $\begin{array}{l}\text { Ekspor_Ind (- } \\
\text { 2) }\end{array}$ & -2.30 & & $\begin{array}{l}\text { Resona_Per } \\
\text { dania }(-4)\end{array}$ & -2.53 & & Maybank (-2) & 2.52 \\
\hline & $\begin{array}{l}\text { Ekspor_Ind (- } \\
\text { 3) }\end{array}$ & 2.34 & $\begin{array}{l}\text { CIMB } \\
\text { NIAGA }\end{array}$ & $\mathrm{BCA}(-2)$ & -2.24 & & Maybank (-3) & -2.58 \\
\hline & $\begin{array}{l}\text { Ekspor_Ind (- } \\
\text { 4) }\end{array}$ & -2.37 & & BCA $(-3)$ & -2.98 & & Maybank (-4) & 2.63 \\
\hline & KEBHana (-2) & -1.7 & & BNI (-1) & -3.04 & & Mega (-1) & -2.35 \\
\hline & KEBHana (-3) & 1.81 & & BRI (-1) & 2.23 & & Mega (-2) & 1.89 \\
\hline & KEBHana (-4) & -1.83 & & BRI (-2) & -2.45 & & Permata $(-3)$ & -1.69 \\
\hline & Mandiri (-4) & -1.68 & & BRI (-3) & -2.13 & & Permata $(-4)$ & 1.9 \\
\hline & Maybank (-1) & 1.98 & & BRI (-4) & -2.62 & & $\begin{array}{l}\text { Woori_SDRA } \\
(-1)\end{array}$ & -2.83 \\
\hline & Maybank (-2) & -2.04 & & BTN (-1) & 3.44 & & $\begin{array}{l}\text { Woori_SDRA } \\
(-2)\end{array}$ & 3.01 \\
\hline & Maybank (-3) & 2.10 & & BTN (-4) & -2.24 & & $\begin{array}{l}\text { Woori_SDRA } \\
(-3)\end{array}$ & -3.18 \\
\hline & Maybank (-4) & -2.16 & & $\begin{array}{l}\text { CIMB_Niag } \\
\text { a }(-1)\end{array}$ & 2.43 & & $\begin{array}{l}\text { Woori_SDRA } \\
(-4)\end{array}$ & 3.32 \\
\hline & Mega (-1) & 2.38 & & $\begin{array}{l}\text { Danamon (- } \\
\text { 4) }\end{array}$ & -1.76 & $\begin{array}{l}\text { JTRUS } \\
\text { T } \\
\text { INDON } \\
\text { ESIA }\end{array}$ & $\mathrm{BCA}(-3)$ & -2.11 \\
\hline & Mega (-2) & -2.07 & & $\begin{array}{l}\text { Ekspor_Ind } \\
(-1)\end{array}$ & 2.66 & & BNI $(-1)$ & -2.501 \\
\hline
\end{tabular}


Musdholifah, Hartono, \& Wulandari

Measuring Contagion Risk on Banking System in The Digital Era

\begin{tabular}{|c|c|c|c|c|c|c|c|c|}
\hline Bank & Bank & Respon & Bank & Bank & Respon & Bank & Bank & Respon \\
\hline & $\begin{array}{l}\text { Woori_SDRA } \\
(-1)\end{array}$ & 2.75 & & $\begin{array}{l}\text { Ekspor_Ind } \\
(-2)\end{array}$ & -2.7 & & BRI (-2) & -3.06 \\
\hline & $\begin{array}{l}\text { Woori_SDRA } \\
(-2)\end{array}$ & -2.87 & & $\begin{array}{l}\text { Ekspor_Ind } \\
(-3)\end{array}$ & 2.74 & & BRI (-3) & -2.72 \\
\hline & $\begin{array}{l}\text { Woori_SDRA } \\
(-3)\end{array}$ & 3.01 & & $\begin{array}{l}\text { Ekspor_Ind } \\
(-4)\end{array}$ & -2.8 & & BRI (-4) & -1.99 \\
\hline & $\begin{array}{l}\text { Woori_SDRA } \\
(-4)\end{array}$ & -3.16 & & $\begin{array}{l}\text { Jtrust_Ind } \\
(-3)\end{array}$ & 1.84 & & BTN (-1) & 4.49 \\
\hline \multirow{25}{*}{$\begin{array}{l}\text { EKSPOR } \\
\text { INDONES } \\
\text { IA }\end{array}$} & $\mathrm{BCA}(-2)$ & -1.7 & & $\begin{array}{l}\text { Jtrust_Ind } \\
(-4)\end{array}$ & -2.11 & & BTN (-4) & -3.52 \\
\hline & BNI $(-1)$ & 2.6 & & $\begin{array}{l}\text { KEBHana (- } \\
\text { 1) }\end{array}$ & 2.16 & & $\begin{array}{l}\text { CIMB Niaga (- } \\
\text { 1) }\end{array}$ & 4.34 \\
\hline & $\begin{array}{l}\text { CIMB Niaga (- } \\
\text { 1) }\end{array}$ & -3.18 & & $\begin{array}{l}\text { KEBHana (- } \\
\text { 2) }\end{array}$ & -2.43 & & $\begin{array}{l}\text { CIMB Niaga (- } \\
\text { 2) }\end{array}$ & 2.63 \\
\hline & $\begin{array}{l}\text { CIMB Niaga (- } \\
\text { 2) }\end{array}$ & -2.67 & & $\begin{array}{l}\text { KEBHana (- } \\
\text { 3) }\end{array}$ & 2.52 & & $\begin{array}{l}\text { CIMB Niaga (- } \\
\text { 3) }\end{array}$ & 2.29 \\
\hline & $\begin{array}{l}\text { CIMB Niaga (- } \\
\text { 3) }\end{array}$ & -2.31 & & $\begin{array}{l}\text { KEBHana (- } \\
4)\end{array}$ & -2.54 & & Danamon (-4) & -1.88 \\
\hline & Danamon (-1) & 1.9 & & Mandiri (-4) & -2.61 & & $\begin{array}{l}\text { Ekspor_Ind (- } \\
\text { 1) }\end{array}$ & 2.09 \\
\hline & Danamon (-2) & 3.36 & & $\begin{array}{l}\text { Mayapada } \\
(-2)\end{array}$ & 1.90 & & $\begin{array}{l}\text { Ekspor_Ind (- } \\
\text { 2) }\end{array}$ & -2.08 \\
\hline & Danamon (-3) & 2.19 & & $\begin{array}{l}\text { Mayapada } \\
(-3)\end{array}$ & -2.12 & & $\begin{array}{l}\text { Ekspor_Ind (- } \\
\text { 3) }\end{array}$ & 2.09 \\
\hline & Jtrust_Ind $(-1)$ & 1.81 & & $\begin{array}{l}\text { Mayapada } \\
(-4)\end{array}$ & 2.31 & & $\begin{array}{l}\text { Ekspor_Ind (- } \\
\text { 4) }\end{array}$ & -2.12 \\
\hline & Jtrust_Ind (-2) & -1.7 & & $\begin{array}{l}\text { Maybank (- } \\
\text { 1) }\end{array}$ & 2.44 & & KEBHana (-1) & -1.68 \\
\hline & Mega (-1) & -2.99 & & $\begin{array}{l}\text { Maybank (- } \\
\text { 2) }\end{array}$ & -2.53 & & KEBHana (-3) & 1.76 \\
\hline & Mega (-2) & 2.77 & & $\begin{array}{l}\text { Maybank (- } \\
\text { 3) }\end{array}$ & 2.64 & & KEBHana (-4) & -1.76 \\
\hline & Mega (-3) & -2.52 & & $\begin{array}{l}\text { Maybank (- } \\
\text { 4) }\end{array}$ & -2.73 & & Maybank (-1) & 1.83 \\
\hline & Mega (-4) & 2.17 & & $\begin{array}{l}\text { Nusantara } \\
\text { Parahy }(-4)\end{array}$ & 1.89 & & Maybank (-2) & -1.87 \\
\hline & $\begin{array}{l}\text { Nusantara_Pa } \\
\text { rahy }(-1)\end{array}$ & -2.27 & & $\begin{array}{l}\text { Permata (- } \\
\text { 2) }\end{array}$ & -1.73 & & Maybank (-3) & 1.91 \\
\hline & $\begin{array}{l}\text { Nusantara_Pa } \\
\text { rahy }(-2)\end{array}$ & 2.09 & & $\begin{array}{l}\text { Permata (- } \\
\text { 3) }\end{array}$ & 2.01 & & Maybank (-4) & -1.94 \\
\hline & $\begin{array}{l}\text { Nusantara_Pa } \\
\text { rahy }(-3)\end{array}$ & -1.85 & & $\begin{array}{l}\text { Permata (- } \\
\text { 4) }\end{array}$ & -2.16 & & $\begin{array}{l}\text { Woori_SDRA } \\
(-2)\end{array}$ & -1.82 \\
\hline & $\begin{array}{l}\text { Resona_Perd } \\
\text { ania }(-2)\end{array}$ & 2.20 & & $\begin{array}{l}\text { Resona_Per } \\
\text { dania }(-4)\end{array}$ & 1.7 & & $\begin{array}{l}\text { Woori_SDRA } \\
(-3)\end{array}$ & 1.96 \\
\hline & $\begin{array}{l}\text { Resona_Perd } \\
\text { ania }(-3)\end{array}$ & -1.99 & & $\begin{array}{l}\text { Woori_SDR } \\
\text { A (-1) }\end{array}$ & 1.99 & & $\begin{array}{l}\text { Woori_SDRA } \\
(-4)\end{array}$ & -2.06 \\
\hline & $\begin{array}{l}\text { Resona_Perd } \\
\text { ania }(-4)\end{array}$ & 1.72 & & $\begin{array}{l}\text { Woori_SDR } \\
\text { A (-2) }\end{array}$ & -2.13 & $\begin{array}{l}\text { KEB } \\
\text { HANA } \\
\text { INDON } \\
\text { ESIA }\end{array}$ & BCA $(-3)$ & 1.67 \\
\hline & $\begin{array}{l}\text { Woori_SDRA } \\
(-1)\end{array}$ & -2.82 & & $\begin{array}{l}\text { Woori_SDR } \\
\text { A (-3) }\end{array}$ & -2.28 & & BRI (-2) & 3.00 \\
\hline & $\begin{array}{l}\text { Woori_SDRA } \\
(-2)\end{array}$ & 2.49 & & $\begin{array}{l}\text { Woori_SDR } \\
\text { A (-4) }\end{array}$ & -2.42 & & BRI (-3) & 3.18 \\
\hline & $\begin{array}{l}\text { Woori_SDRA } \\
(-3)\end{array}$ & -2.11 & $\begin{array}{l}\text { DANAM } \\
\text { ON }\end{array}$ & BNI (-1) & 2.56 & & BRI (-4) & 1.95 \\
\hline & $\begin{array}{l}\text { Woori_SDRA } \\
(-4)\end{array}$ & 1.77 & & BRI (-3) & 2.15 & & $\begin{array}{l}\text { Commonweal } \\
\text { th }(-1)\end{array}$ & -1.96 \\
\hline & C & -2.29 & & $\begin{array}{l}\text { Danamon } \\
(-1)\end{array}$ & -1.83 & & $\begin{array}{l}\text { Commonweal } \\
\text { th }(-2)\end{array}$ & 1.912 \\
\hline MANDIRI & BCA (-2) & 1.96 & & $\begin{array}{l}\text { Ekspor_Ind } \\
(-1)\end{array}$ & -2.41 & & $\begin{array}{l}\text { Commonweal } \\
\text { th }(-3)\end{array}$ & -1.89 \\
\hline
\end{tabular}


Musdholifah, Hartono, \& Wulandari

Measuring Contagion Risk on Banking System in The Digital Era

\begin{tabular}{|c|c|c|c|c|c|c|c|c|}
\hline Bank & Bank & Respon & Bank & Bank & Respon & Bank & Bank & Respon \\
\hline & $\mathrm{BCA}(-3)$ & 2.82 & & $\begin{array}{l}\text { Ekspor_Ind } \\
(-2)\end{array}$ & 2.56 & & $\begin{array}{l}\text { Commonweal } \\
\text { th }(-4)\end{array}$ & 1.9 \\
\hline & $\operatorname{BRI}(-1)$ & -2.19 & & $\begin{array}{l}\text { Ekspor_Ind } \\
(-3)\end{array}$ & -2.67 & & $\begin{array}{l}\text { Ekspor_Ind (- } \\
\text { 1) }\end{array}$ & -3.22 \\
\hline & BRI (-2) & 3.01 & & $\begin{array}{l}\text { Ekspor_Ind } \\
(-4)\end{array}$ & 2.75 & & $\begin{array}{l}\text { Ekspor_Ind (- } \\
\text { 2) }\end{array}$ & 3.36 \\
\hline & BRI (-3) & 2.78 & & $\begin{array}{l}\text { Jtrust_Ind } \\
(-1)\end{array}$ & -2.88 & & $\begin{array}{l}\text { Ekspor_Ind (- } \\
\text { 3) }\end{array}$ & -3.48 \\
\hline & BRI (-4) & 2.57 & & $\begin{array}{l}\text { Jtrust_Ind } \\
(-2)\end{array}$ & 3.05 & & $\begin{array}{l}\text { Ekspor_Ind (- } \\
\text { 4) }\end{array}$ & 3.578 \\
\hline & BTN $(-1)$ & -3.31 & & $\begin{array}{l}\text { Jtrust_Ind } \\
(-3)\end{array}$ & -3.11 & & Jtrust_Ind (-1) & -2.33 \\
\hline & BTN (-4) & 2.52 & & $\begin{array}{l}\text { Jtrust_Ind } \\
(-4)\end{array}$ & 3.12 & & Jtrust_Ind (-2) & 2.75 \\
\hline & $\begin{array}{l}\text { CIMB Niaga (- } \\
\text { 1) }\end{array}$ & -2.81 & & $\begin{array}{l}\text { KEBHana (- } \\
\text { 1) }\end{array}$ & -2.93 & & Jtrust_Ind (-3) & -3.02 \\
\hline & $\begin{array}{l}\text { CIMB Niaga (- } \\
\text { 2) }\end{array}$ & -2.06 & & $\begin{array}{l}\text { KEBHana (- } \\
\text { 2) }\end{array}$ & 2.93 & & Jtrust_Ind (-4) & 3.21 \\
\hline & $\begin{array}{l}\text { Commonweal } \\
\text { th }(-1)\end{array}$ & -2.01 & & $\begin{array}{l}\text { KEBHana (- } \\
\text { 3) }\end{array}$ & -2.95 & & KEBHana $(-1)$ & -3.23 \\
\hline & $\begin{array}{l}\text { Commonweal } \\
\text { th }(-2)\end{array}$ & 1.84 & & $\begin{array}{l}\text { KEBHana (- } \\
4)\end{array}$ & 2.95 & & KEBHana (-2) & 3.43 \\
\hline & $\begin{array}{l}\text { Commonweal } \\
\text { th }(-3)\end{array}$ & -1.71 & & Mandiri (-4) & 1.96 & & KEBHana (-3) & -3.48 \\
\hline & $\begin{array}{l}\text { Ekspor_Ind (- } \\
\text { 1) }\end{array}$ & -3.04 & & $\begin{array}{l}\text { Mayapada } \\
(-1)\end{array}$ & 3.01 & & KEBHana (-4) & 3.48 \\
\hline & $\begin{array}{l}\text { Ekspor_Ind (- } \\
\text { 2) }\end{array}$ & 3.05 & & $\begin{array}{l}\text { Mayapada } \\
(-2)\end{array}$ & -3.09 & & Mandiri (-2) & 1.77 \\
\hline & $\begin{array}{l}\text { Ekspor_Ind (- } \\
\text { 3) }\end{array}$ & -3.07 & & $\begin{array}{l}\text { Mayapada } \\
(-3)\end{array}$ & 3.1 & & Mandiri (-4) & 2.53 \\
\hline & $\begin{array}{l}\text { Ekspor_Ind (- } \\
\text { 4) }\end{array}$ & 3.10 & & $\begin{array}{l}\text { Mayapada } \\
(-4)\end{array}$ & -3.1 & & $\begin{array}{l}\text { Mayapada (- } \\
\text { 1) }\end{array}$ & 2.78 \\
\hline & Jtrust_Ind (-4) & 1.89 & & $\begin{array}{l}\text { Maybank (- } \\
\text { 1) }\end{array}$ & -2.07 & & $\begin{array}{l}\text { Mayapada (- } \\
\text { 2) }\end{array}$ & -3.08 \\
\hline & KEBHana (-1) & -2.24 & & $\begin{array}{l}\text { Maybank (- } \\
\text { 2) }\end{array}$ & 2.22 & & $\begin{array}{l}\text { Mayapada (- } \\
\text { 3) }\end{array}$ & 3.24 \\
\hline & KEBHana (-2) & 2.54 & & $\begin{array}{l}\text { Maybank (- } \\
\text { 3) }\end{array}$ & -2.36 & & $\begin{array}{l}\text { Mayapada (- } \\
\text { 4) }\end{array}$ & -3.35 \\
\hline & KEBHana (-3) & -2.62 & & $\begin{array}{l}\text { Maybank (- } \\
\text { 4) }\end{array}$ & 2.46 & & Maybank (-1) & -3.01 \\
\hline & KEBHana (-4) & 2.60 & & Mega (-2) & -1.96 & & Maybank (-2) & 3.16 \\
\hline & Mandiri (-4) & 2.131 & & Mega (-3) & 2.40 & & Maybank (-3) & -3.32 \\
\hline & $\begin{array}{l}\text { Mayapada (- } \\
\text { 2) }\end{array}$ & -1.69 & & Mega (-4) & -2.68 & & Maybank (-4) & 3.44 \\
\hline & $\begin{array}{l}\text { Mayapada (- } \\
\text { 3) }\end{array}$ & 1.98 & & $\begin{array}{l}\text { Nusantara_- } \\
\text { Parahy }(-1)\end{array}$ & 2.59 & & Mega (-4) & -1.76 \\
\hline & $\begin{array}{l}\text { Mayapada (- } \\
\text { 4) }\end{array}$ & -2.20 & & $\begin{array}{l}\text { Nusantara } \\
\text { Parahy }(-2)\end{array}$ & -2.87 & & $\begin{array}{l}\text { Nusantara_Pa } \\
\text { rahy }(-1)\end{array}$ & 1.72 \\
\hline & Maybank (-1) & -2.88 & & $\begin{array}{l}\text { Nusantara } \\
\text { Parahy }(-3)\end{array}$ & 3.03 & & $\begin{array}{l}\text { Nusantara_Pa } \\
\text { rahy }(-2)\end{array}$ & -2.29 \\
\hline & Maybank (-2) & 2.95 & & $\begin{array}{l}\text { Nusantara_- } \\
\text { Parahy (-4) }\end{array}$ & -3.11 & & $\begin{array}{l}\text { Nusantara_Pa } \\
\text { rahy }(-3)\end{array}$ & 2.73 \\
\hline & Maybank (-3) & -3.01 & & $\begin{array}{l}\text { Permata (- } \\
\text { 1) }\end{array}$ & -2.96 & & $\begin{array}{l}\text { Nusantara_Pa } \\
\text { rahy }(-4)\end{array}$ & -3.05 \\
\hline & Maybank (-4) & 3.05 & & $\begin{array}{l}\text { Permata (- } \\
\text { 2) }\end{array}$ & 3.09 & & Permata (-1) & -2.46 \\
\hline & Mega (-1) & -2.18 & & $\begin{array}{l}\text { Permata (- } \\
\text { 3) }\end{array}$ & -3.10 & & Permata (-2) & 2.95 \\
\hline & Mega (-2) & 1.74 & & $\begin{array}{l}\text { Permata (- } \\
\text { 4) }\end{array}$ & 3.085 & & Permata $(-3)$ & -3.15 \\
\hline & Permata $(-3)$ & -1.80 & & $\begin{array}{l}\text { Resona_Per } \\
\text { dania }(-\overline{2})\end{array}$ & -2.83 & & Permata $(-4)$ & 3.25 \\
\hline
\end{tabular}


Musdholifah, Hartono, \& Wulandari

Measuring Contagion Risk on Banking System in The Digital Era

\begin{tabular}{|c|c|c|c|c|c|c|c|c|}
\hline Bank & Bank & Respon & Bank & Bank & Respon & Bank & Bank & Respon \\
\hline & Permata (-4) & 2.01 & & $\begin{array}{l}\text { Resona_Per } \\
\text { dania }(-3)\end{array}$ & 3.01 & & $\begin{array}{l}\text { Resona_Perd } \\
\text { ania }(-2)\end{array}$ & -2.05 \\
\hline & $\begin{array}{l}\text { Woori_SDRA } \\
(-1)\end{array}$ & -3.01 & & $\begin{array}{l}\text { Resona_Per } \\
\text { dania }(-4)\end{array}$ & -3.08 & & $\begin{array}{l}\text { Resona_Perd } \\
\text { ania }(-3)\end{array}$ & 2.58 \\
\hline & $\begin{array}{l}\text { Woori_SDRA } \\
(-2)\end{array}$ & 3.17 & $\begin{array}{l}\text { MAYBA } \\
\text { NK } \\
\text { INDONE } \\
\text { SIA }\end{array}$ & BNI $(-1)$ & -5.06 & & $\begin{array}{l}\text { Resona_Perd } \\
\text { ania }(-4)\end{array}$ & -2.90 \\
\hline & $\begin{array}{l}\text { Woori_SDRA } \\
(-3)\end{array}$ & -3.34 & & BNI (-2) & -2.62 & & $\begin{array}{l}\text { Woori_SDRA } \\
(-3)\end{array}$ & -1.88 \\
\hline & $\begin{array}{l}\text { Woori_SDRA } \\
(-4)\end{array}$ & 3.51 & & BNI (-3) & -2.54 & & $\begin{array}{l}\text { Woori_SDRA } \\
(-4)\end{array}$ & 2.11 \\
\hline & $\mathrm{C}$ & -1.85 & & BTN $(-1)$ & 3.15 & MEGA & $\mathrm{BCA}(-2)$ & -1.82 \\
\hline & BNI (-1) & -2.01 & & $\begin{array}{l}\text { CIMB Niaga } \\
(-1)\end{array}$ & 4.46 & & $\mathrm{BCA}(-3)$ & -2.55 \\
\hline & BTN (-1) & 2.59 & & $\begin{array}{l}\text { CIMB Niaga } \\
(-2)\end{array}$ & 2.85 & & BRI $(-2)$ & -2.19 \\
\hline & BTN (-4) & -1.68 & & $\begin{array}{l}\text { CIMB Niaga } \\
(-3)\end{array}$ & 2.50 & & BTN $(-1)$ & 4.52 \\
\hline & $\begin{array}{l}\text { CIMB Niaga (- } \\
\text { 1) }\end{array}$ & 2.89 & & $\begin{array}{l}\text { Danamon (- } \\
\text { 2) }\end{array}$ & -1.87 & & BTN (-2) & -1.77 \\
\hline & $\begin{array}{l}\text { CIMB Niaga (- } \\
\text { 2) }\end{array}$ & 1.92 & & $\begin{array}{l}\text { Danamon (- } \\
\text { 4) }\end{array}$ & -2.14 & & BTN (-4) & -3.29 \\
\hline & $\begin{array}{l}\text { CIMB Niaga (- } \\
\text { 3) }\end{array}$ & 1.76 & & $\begin{array}{l}\text { Resona_Per } \\
\text { dania }(-1)\end{array}$ & -1.84 & & $\begin{array}{l}\text { CIMB Niaga (- } \\
\text { 1) }\end{array}$ & 2.53 \\
\hline \multirow[t]{18}{*}{$\begin{array}{l}\text { NUSANT } \\
\text { ARA } \\
\text { PARAH- } \\
\text { YANGAN }\end{array}$} & $\mathrm{BCA}(-3)$ & -1.99 & $\begin{array}{l}\text { RESON } \\
\text { A } \\
\text { PERDA } \\
\text { NIA }\end{array}$ & $\begin{array}{l}\text { Jtrust_Ind } \\
(-1)\end{array}$ & 4.22 & & $\begin{array}{l}\text { CIMB Niaga (- } \\
\text { 2) }\end{array}$ & 3.18 \\
\hline & BNI (-1) & -3.33 & & $\begin{array}{l}\text { Jtrust_Ind } \\
(-2)\end{array}$ & -3.66 & & $\begin{array}{l}\text { CIMB Niaga (- } \\
\text { 3) }\end{array}$ & 2.54 \\
\hline & BRI (-1) & 2.05 & & $\begin{array}{l}\text { Jtrust_Ind } \\
(-3)\end{array}$ & 3.23 & & $\begin{array}{l}\text { CIMB Niaga (- } \\
4)\end{array}$ & 1.95 \\
\hline & BRI (-2) & -3.38 & & $\begin{array}{l}\text { Jtrust_Ind } \\
(-4)\end{array}$ & -2.89 & & Danamon $(-2)$ & -2.38 \\
\hline & BRI (-3) & -3.29 & & $\begin{array}{l}\text { KEBHana (- } \\
\text { 1) }\end{array}$ & 2.31 & & Jtrust_Ind $(-1)$ & -1.83 \\
\hline & BRI (-4) & -2.59 & & $\begin{array}{l}\text { KEBHana (- } \\
\text { 2) }\end{array}$ & -1.92 & & Mega (-1) & 4.12 \\
\hline & BTN (-1) & 3.74 & & $\begin{array}{l}\text { KEBHana (- } \\
3)\end{array}$ & 1.78 & & Mega (-2) & -3.84 \\
\hline & BTN (-4) & -2.89 & & $\begin{array}{l}\text { KEBHana (- } \\
\text { 4) }\end{array}$ & -1.75 & & Mega (-3) & 3.35 \\
\hline & $\begin{array}{l}\text { CIMB Niaga (- } \\
\text { 1) }\end{array}$ & 3.73 & & $\begin{array}{l}\text { Mayapada } \\
(-1)\end{array}$ & -3.60 & & Mega (-4) & -2.65 \\
\hline & $\begin{array}{l}\text { CIMB Niaga (- } \\
\text { 2) }\end{array}$ & 1.72 & & $\begin{array}{l}\text { Mayapada } \\
(-2)\end{array}$ & 3.09 & & $\begin{array}{l}\text { Nusantara_Pa } \\
\text { rahy }(-1)\end{array}$ & 2.48 \\
\hline & Danamon (-4) & -2.03 & & $\begin{array}{l}\text { Mayapada } \\
(-3)\end{array}$ & -2.75 & & $\begin{array}{l}\text { Nusantara_Pa } \\
\text { rahy }(-2)\end{array}$ & -1.88 \\
\hline & $\begin{array}{l}\text { Ekspor_Ind (- } \\
\text { 1) }\end{array}$ & 3.45 & & $\begin{array}{l}\text { Mayapada } \\
(-4)\end{array}$ & 2.52 & & Permata $(-1)$ & -1.76 \\
\hline & $\begin{array}{l}\text { Ekspor_Ind (- } \\
\text { 2) }\end{array}$ & -3.51 & & Mega (-1) & -4.92 & & $\begin{array}{l}\text { Resona_Perd } \\
\text { ania }(-1)\end{array}$ & 2.72 \\
\hline & $\begin{array}{l}\text { Ekspor_Ind (- } \\
\text { 3) }\end{array}$ & 3.57 & & Mega (-2) & 5.09 & & $\begin{array}{l}\text { Resona_Perd } \\
\text { ania }(-2)\end{array}$ & -2.49 \\
\hline & $\begin{array}{l}\text { Ekspor_Ind (- } \\
\text { 4) }\end{array}$ & -3.63 & & Mega (-3) & -4.85 & & $\begin{array}{l}\text { Woori_SDRA } \\
(-1)\end{array}$ & 3.63 \\
\hline & Jtrust_Ind $(-1)$ & 2.02 & & Mega (-4) & 4.27 & & $\begin{array}{l}\text { Woori_SDRA } \\
(-2)\end{array}$ & -3.53 \\
\hline & Jtrust_Ind (-2) & -2.47 & & $\begin{array}{l}\text { Nusantara } \\
\text { Parahy (-1) }\end{array}$ & -4.62 & & $\begin{array}{l}\text { Woori_SDRA } \\
(-3)\end{array}$ & 3.41 \\
\hline & Jtrust_Ind (-3) & 2.79 & & $\begin{array}{l}\text { Nusantara } \\
\text { Parahy }(-2)\end{array}$ & 4.09 & & $\begin{array}{l}\text { Woori_SDRA } \\
(-4)\end{array}$ & -3.37 \\
\hline
\end{tabular}


Musdholifah, Hartono, \& Wulandari

Measuring Contagion Risk on Banking System in The Digital Era

\begin{tabular}{|c|c|c|c|c|c|c|c|c|}
\hline Bank & Bank & Respon & Bank & Bank & Respon & Bank & Bank & Respon \\
\hline & Jtrust_Ind (-4) & -3.04 & & $\begin{array}{l}\text { Nusantara_ } \\
\text { Parahy }(-3)\end{array}$ & -3.62 & $\begin{array}{l}\text { PERM } \\
\text { ATA }\end{array}$ & BNI (-1) & -4.69 \\
\hline & KEBHana (-1) & 3.04 & & $\begin{array}{l}\text { Nusantara } \\
\text { Parahy }(-4)\end{array}$ & 3.19 & & BNI (-3) & -2.01 \\
\hline & KEBHana (-2) & -3.29 & & $\begin{array}{l}\text { Permata (- } \\
\text { 1) }\end{array}$ & 4.06 & & $\begin{array}{l}\text { CIMB Niaga (- } \\
\text { 1) }\end{array}$ & 1.82 \\
\hline & KEBHana $(-3)$ & -3.36 & & $\begin{array}{l}\text { Permata (- } \\
\text { 2) }\end{array}$ & -3.37 & & $\begin{array}{l}\text { Resona_Perd } \\
\text { ania }(-1)\end{array}$ & -1.83 \\
\hline & KEBHana (-4) & -3.36 & & $\begin{array}{l}\text { Permata (- } \\
\text { 3) }\end{array}$ & 2.94 & $\begin{array}{l}\text { WOOR } \\
\text { I } \\
\text { SAUDA } \\
\text { RA }\end{array}$ & $\begin{array}{l}\text { Ekspor_Ind (- } \\
\text { 2) }\end{array}$ & 1.69 \\
\hline & Mandiri (-2) & -1.80 & & $\begin{array}{l}\text { Permata (- } \\
\text { 4) }\end{array}$ & -2.67 & & $\begin{array}{l}\text { Ekspor_Ind (- } \\
\text { 3) }\end{array}$ & -1.76 \\
\hline & Mandiri (-4) & -2.56 & & $\begin{array}{l}\text { Resona_Per } \\
\text { dania }(-1)\end{array}$ & -5.11 & & $\begin{array}{l}\text { Ekspor_Ind (- } \\
\text { 4) }\end{array}$ & 1.81 \\
\hline & $\begin{array}{l}\text { Mayapada (- } \\
\text { 1) }\end{array}$ & -2.57 & & $\begin{array}{l}\text { Resona_Per } \\
\text { dania }(-2)\end{array}$ & 4.57 & & Mandiri (-4) & 1.67 \\
\hline & $\begin{array}{l}\text { Mayapada (- } \\
\text { 2) }\end{array}$ & 2.87 & & $\begin{array}{l}\text { Resona_Per } \\
\text { dania }(-3)\end{array}$ & -3.89 & & $\begin{array}{l}\text { Woori_SDRA } \\
(-3)\end{array}$ & -1.69 \\
\hline & $\begin{array}{l}\text { Mayapada (- } \\
\text { 3) }\end{array}$ & -3.07 & & $\begin{array}{l}\text { Resona_Per } \\
\text { dania }(-4)\end{array}$ & 3.35 & & $\begin{array}{l}\text { Woori_SDRA } \\
(-4)\end{array}$ & 1.79 \\
\hline & $\begin{array}{l}\text { Mayapada (- } \\
\text { 4) }\end{array}$ & 3.23 & & $\begin{array}{l}\text { Woori_SDR } \\
\text { A }(-1)\end{array}$ & -3.72 & & & \\
\hline & Maybank (-1) & 3.06 & & $\begin{array}{l}\text { Woori_SDR } \\
\text { A (-2) }\end{array}$ & 3.44 & & & \\
\hline & Maybank (-2) & -3.17 & & $\begin{array}{l}\text { Woori_SDR } \\
\text { A (-3) }\end{array}$ & -3.17 & & & \\
\hline & Maybank (-3) & 3.27 & & $\begin{array}{l}\text { Woori_SDR } \\
\text { A (-4) }\end{array}$ & 2.98 & & & \\
\hline & Maybank (-4) & -3.34 & & & & & & \\
\hline & $\begin{array}{l}\text { Nusantara_Pa } \\
\text { rahy }(-2)\end{array}$ & 1.95 & & & & & & \\
\hline & $\begin{array}{l}\text { Nusantara_Pa } \\
\text { rahy }(-3)\end{array}$ & -2.46 & & & & & & \\
\hline & $\begin{array}{l}\text { Nusantara_Pa } \\
\text { rahy }(-4)\end{array}$ & 2.83 & & & & & & \\
\hline & Permata $(-1)$ & 2.08 & & & & & & \\
\hline & Permata (-2) & -2.69 & & & & & & \\
\hline & Permata (-3) & 2.96 & & & & & & \\
\hline & Permata (-4) & -3.07 & & & & & & \\
\hline & $\begin{array}{l}\text { Resona_Perd } \\
\text { ania }(-3)\end{array}$ & -2.26 & & & & & & \\
\hline & $\begin{array}{l}\text { Resona_Perd } \\
\text { ania }(-4)\end{array}$ & 2.60 & & & & & & \\
\hline & $\begin{array}{l}\text { Woori_SDRA } \\
(-1)\end{array}$ & 1.88 & & & & & & \\
\hline & $\begin{array}{l}\text { Woori_SDRA } \\
(-2)\end{array}$ & -2.11 & & & & & & \\
\hline & $\begin{array}{l}\text { Woori_SDRA } \\
(-3)\end{array}$ & 2.34 & & & & & & \\
\hline & $\begin{array}{l}\text { Woori_SDRA } \\
(-4)\end{array}$ & -2.51 & & & & & & \\
\hline & $\mathrm{C}$ & 2.04 & & & & & & \\
\hline
\end{tabular}

\section{References}

Aharony, J., \& Swary, I. (1983). Contagion Effects of Bank Failures: Evidence from Capital Markets. The Journal of Business, 56(3), 305-322. https://doi.org/10.1086/296203 


\section{Musdholifah, Hartono, \& Wulandari \\ Measuring Contagion Risk on Banking System in The Digital Era}

Ahelegbey, D. F., \& Giudici, P. (2014). Bayesian Selection of Systemic Risk Networks. Bayesian Model Comaprison Advances in Econometrics, 34, 117-153. https://doi.org/10.1108/s0731-905320140000034007

Allen, F., \& Gale, D. (2000). Financial Contagion. Journal of Political Economy, 108(1), 1-33. https://doi.org/10.1086/262109

Allen, F., Carletti, E., \& Gale, D. (2009). Interbank Market Liquidity and Central Bank Intervention. Journal of Monetary Economics, 56(5), 639-652. https://doi.org/10.1016/j.jmoneco.2009.04.003

Ayomi, S., \& Hermanto, B. (2013). Mengukur Risiko Sistemik dan Keterkaitan Finansial Perbankan di Indonesia. Buletin Ekonomi Moneter dan Perbankan, 103-126.

Billio, M., Getmansky, M., Lo, A. W., \& Pelizzon, L. (2010). Measuring Systemic Risk in the Finance and Insurance Sectors. MIT Sloan School Working Paper 4774-10. Retrieved from http://ssrn.com/abstract=1573902

Chen, H., Chen, Q., \& Gerlach, S. (2013). The Implementation of Monetary Policy in China: The Interbank Market and Bank Lending. Global Banking, Financial Markets and Crises (International Finance Review, 14). 31-69. https://doi.org/10.1108/S15693767(2013)0000014005

Chinazzi, M., \& Fagiolo, G. (2013). Systemic Risk, Contagion, and Financial Networks: A Survey. LEM Working Paper Series, No. 2013/08. Retrieved from https://www.econstor.eu/bitstream/10419/89353/1/740929119.pdf

Christiawan, N. G., \& Arfianto, E. D. (2013). Interbank Contagious : Sistemik Market Risk Kasus pada Perbankan Indonesia. Jurnal Studi Manajemen dan Organisasi, 10(1), 38-48. https://doi.org/10.14710/ismo.v10i1.5575

De Bandt, O., \& Hartmann, P. (2000). Systemic Risk: A Survey. European Central Bank Working Paper Series. Retrieved from https://www.ecb.europa.eu/pub/pdf/scpwps/ecbwp035.pdf

Gai, P., Haldane, A., \& Kapadia, S. (2011). Complexity , concentration and contagion. Journal of Monetary Economics, 58(5), 453-470. https://doi.org/10.1016/j.jmoneco.2011.05.005

Gai, P., Jenkinson, N., \& Kapadia, S. (2007). Systemic Risk in Modern Financial Systems: Analytics and Policy Design. Journal of Risk Finance, 8(2), 156-165. https://doi.org/10.1108/15265940710732387

Gauthier, C., He, Z., \& Souissi, M. (2010). Understanding Systemic Risk: The Trade-Offs between Capital, Short-Term Funding and Liquid Asset Holdings. Bank of Canada Working Paper 2010-29. Retrieved from https://www.bankofcanada.ca/wpcontent/uploads/2010/11/wp10-29.pdf

Grais, W., \& Rajhi, W. (2015). Islamic Finance, Contagion Effects, Spillovers and Monetary Policy. Journal of Islamic Accounting and Business Research, 6(2), 208-221. https://doi.org/10.1108/JIABR-12-2012-0079

Kapoor, S. (2010). The Financial Crisis - Causes \& Cures. In FES Brüssel Bertelsmannstiftung ETUI (pp. 19-20). Brussels: FES Brüssel Bertelsmannstiftung ETUI.

Memmel, C., \& Sachs, A. (2013). Contagion in the Interbank Market and its Determinants. Journal of Financial Stability, 9, 46-54. https://doi.org/10.1016/j.jfs.2013.01.001

Philippas, D., Koutelidakis, Y., \& Leontitsis, A. (2015). Insights into European Interbank Network Contagion. Managerial Finance, 41(8). https://doi.org/10.1108/mf-03-2014$\underline{0095}$

Schoenmaker, D. (1996). Contagion Risk in Banking. Ministry of Finance, The Netherlands. Retrieved from https://web.actuaries.ie/sites/default/files/ermresources/345 contagion risk in banking.pdf 
Shah, A. K. (1997). Analysing Systemic Risk in Banking and Financial Markets. Journal of Financial Regulation and Compliance, 5(1), 37-48. https://doi.org/10.1108/eb024903

Simorangkir, I. (2011). Penyebab Bank Runs di Indonesia : Bad Luck atau Fundamental? Buletin Ekonomi Moneter dan Perbankan 14(1), 51-78. https://doi.org/10.21098/bemp.v14i1.456

Upper, C. (2011). Simulation Methods to Assess the Danger of Contagion in Interbank Markets. Journal of Financial Stability, 7, 111-125. https://doi.org/10.1016/i.jfs.2010.12.001

Zakaria, F. (2015). Systemic Risk and Financial Contagion in Morocco: New Approaches of Quantification. In Overlaps of Private Sector with Public Sector around the Globe, 141-171. https://doi.org/http://dx.doi.org/10.1108/S0196-382120150000031007 